\title{
Cutaneous hemorrhagic bullae in a patient with COVID-19: A case report
}

\author{
Mohammad Karimi Alavije ${ }^{1}$, Mohammad Hadi Karbalaie Niya ${ }^{2,3}$, Afsaneh Sadeghzadeh-Bazargan ${ }^{4}$, Mehdi Nikkhah², \\ Amirhossein Faraji², Nima Motamed ${ }^{5}$, Fahimeh Safarnezhad Tameshkel ${ }^{2}$, Farhad Zamani*2 (D) \\ Received: 25 Jul 2020 \\ Published: 1 Feb 2021
}

\begin{abstract}
COVID-19 was first discovered in Wuhan, China, and has spread rapidly around the world. The most important manifestation of COVID-19 was ARDS-like lung injury at first, but the involvement of other organs, such as kidney, heart, liver, and skin, was gradually reported. It is important to report and share all atypical manifestations of this disease to help other physicians to gain more knowledge about this new viral disease. As mentioned, there are also studies that show different types of cutaneous involvement in these patients, but due to the lack of more detailed studies in this field, and on the other hand, the possible usefulness of skin lesions as a diagnostic or alarming sign in the COVID-19 era, in this study we report a COVID-19 patient with a large hemorrhagic blister similar to sepsis-induced skin lesion. Despite the lack of common symptoms of the disease, the lung scan of the patient was positive for COVID-19.
\end{abstract}

Keywords: COVID-19, Skin lesion, Extra pulmonary complication, Cutaneous manifestation, Coagulopathy, Hemorrhagic bullae, Vascular injury, Vasculitis, Vasculopathy

\author{
Conflicts of Interest: None declared \\ Funding: Iran University of Medical Sciences, Tehran, Iran \\ *This work has been published under CC BY-NC-SA 1.0 license.
Copyright $\subseteq$ Iran University of Medical Sciences
}

Cite this article as: Karimi Alavije M, Karbalaie Niya MH, Sadeghzadeh-Bazargan A, Nikkhah M, Faraji A, Motamed N, Safarnezhad Tameshkel F, Zamani F. Cutaneous hemorrhagic bullae in a patient with COVID-19: A case report. Med J Islam Repub Iran. 2021 (1 Feb);35:15. https://doi.org/10.47176/mjiri.35.15

\section{Introduction}

The novel coronavirus, first identified in Wuhan, China, was named as COVID-19 by the World Health Organization on February 11, 2020 (1). The clinical spectrum of COVID-19 ranges from asymptomatic to symptomatic forms with subclinical or severe respiratory failure that require special care and include systemic sepsis, septic shock, and various syndromes involved in the functioning of the body. Common clinical symptoms include fever, cough, shortness of breath, fatigue, normal or decreased leukocytes, and pneumonia seen on radiographs. Other less common symptoms include headache, sore throat, and rhinorrhea (2). A small number of patients may experience nausea and diarrhea; and symptoms that are more alarming need special care immediately after onset, which include persistent chest pain or pressure, breathing problems, confusion and bruising of the lips or face, and ad- vanced conditions that lead to pneumonia (3). The symptoms of the disease are similar in some cases. However, studies have shown that patients sometimes present with completely different symptoms and even with the symptoms of other diseases (4), and the doctor accidentally notices an involvement in lungs in patients with COVID$19(5,6)$, Also, in this disease, early diagnosis of suspicious people and the patient makes it possible to prevent further spread of the disease by timely treatment and discontinuation of the transmission chain.

\section{Case presentation}

A 68-year-old diabetic woman came to our center because of an asymptomatic ecchymotic patch that suddenly appeared on her lower left limb. Except for diabetes, the patient did not have any history of other underlying dis-

Corresponding author: Dr Farhad Zamani, zamani.f@iums.ac.ir

1. Department of Infectious Diseases, Imam Reza Hospital, Amol, Iran

2. Gastrointestinal and Liver Diseases Research Center, Iran University of Medical Sciences, Tehran, Iran

3. Department of Virology, Iran University of Medical Sciences, Tehran, Iran

4. Department of Dermatology, Rasoul Akram Hospital, Iran University of Medical Sciences, Tehran, Iran

5. Department of Social Medicine, Zanjan University of Medical Sciences, Zanjan, Iran 
ease or medication. The patient's diabetes was controlled by the diet and the patient did not receive any medication for it.

At the time of the first visit, our patient did not have any constitutional symptoms and her vital signs were stable. (blood pressure (BP): 90/60 $\mathrm{mmHg}$; respiration rate (RR): 18 breathes per minute, pulse rate (PR): 78 beats per minute; body temperature: $36.7^{\circ} \mathrm{c}$, and $\mathrm{O}_{2}$ saturation: $97 \%$ ). The dorsalis pedis and posterior tibial pulses were bilaterally palpable. Due to diabetes history, the patient admitted with a coagulopathic-thrombotic impression for more evaluations. During hospitalization, ecchymotic lesion progressed to hemorrhagic bullae with erythematous border without additional symptoms (Fig. 1).

The physician first requested a complete laboratory examination by blood sampling, arterial and venous Doppler ultrasound, CT scan of the lungs, and ECG every 48 hours. In the laboratory studies, white blood cells count (WBC): 20600 with 84.2\% PMN, platelets (PLT): 137 000, hemoglobin $(\mathrm{Hb})$ : 11.9, plasma levels of IgA: 475 mg/dL, IL-6: 60 pg/mL, Amylase: 145 U/L, PRO-BNP: 297 pg/mL, ESR: 41, LDH: 983, PT: 15.5, PTT: 30, INR: 1.3, and BS: 526. The results of liver function test, BUN, $\mathrm{Cr}, \mathrm{CPK}$, were in the normal range, and the results for troponin and CRP and troponin were negative. Also, the results of the patient's 24-hour urine culture test and 24hour and 48-hour blood culture tests were negative for any bacteria.

Doppler sonography reported atheromatous plaque in the right common femoral artery, but the arterial and venous flow of the left lower limb was normal. Differential diagnoses for this skin lesion were as follows:

1. Because of diabetes, necrotizing fasciitis must be considered. However, these patients often have a bad general condition with very severe pain at the site of the involvement, which is not very consistent with the findings of our patient.

2. Coagulation disorders are due to infections and sepsis, such as disseminated intravascular coagulation (DIC) and purpura fulminans (PF), but the sepsis workup of our patient was negative except for chest CT scan findings that will be mentioned later.

3. Drug-induced necrosis and vaso-occlusion, such as heparin and warfarin necrosis, but the drug history of our patient was negative.

4. Vasculitis and vasculopathy are due to other underlying conditions, such as rheumatologic disorders or uncommon infections.

Chest CT scan showed typical bilateral peripheral ground-glass opacities that were more predominant in lower lobes. However, SARS-CoV-2 RT-PCR was negative at the beginning of illness, so COVID-19 diagnosis was based on chest CT imaging (Fig. 2). The only symptom of the patient was fever (38.8 one day after hospitalization), anorexia, and nausea without anosmia. The patient was hospitalized 11 days and underwent a standard national treatment protocol (Lopinavir/ritonavi (Kaletra) + Chloroquine + Ribavirin). Additionally, ceftriaxone, linezolid, ReciGen, and Remicade were prescribed for the patient.

During the hospitalization, the patient did not have any internal or visceral bleeding and involvement. Also, due to high blood glucose, the patient had diabetic diet, and her wound was washed, and she was dressed regularly and daily. Two weeks after the infection, the skin lesion was changed to a necrotic ulcer and was finally healed with minimal scarring.

\section{Discussion}

In this study, the patient was a 68-year-old woman who had no common symptoms of COVID-19 and had only fever, anorexia, and nausea. She was initially visited with coagulation disorder and extensive sepsis bleeding, and COVID-19 disease was diagnosed for her during the additional examinations through a lung scan. So far, there have been few reports of skin lesions resulted by COVID-19 worldwide and in Italy (7), which was one of the centers of the disease. In Recalcati et al study on COVID-19 patients, there were a number of patients with skin manifestations like erythematous rash, extensive urticaria, rashes such as chickenpox and sometimes pruritus after hospitalization. The lesions usually healed within a few days. Studies at Medical Center (UNMC) at the Department of Dermatology of University of Nebraska showed that the existence of the skin rashes in $0.2 \%$ to $1.2 \%$ of the 1099 patients with COVID-19 in the laboratory by January 29,

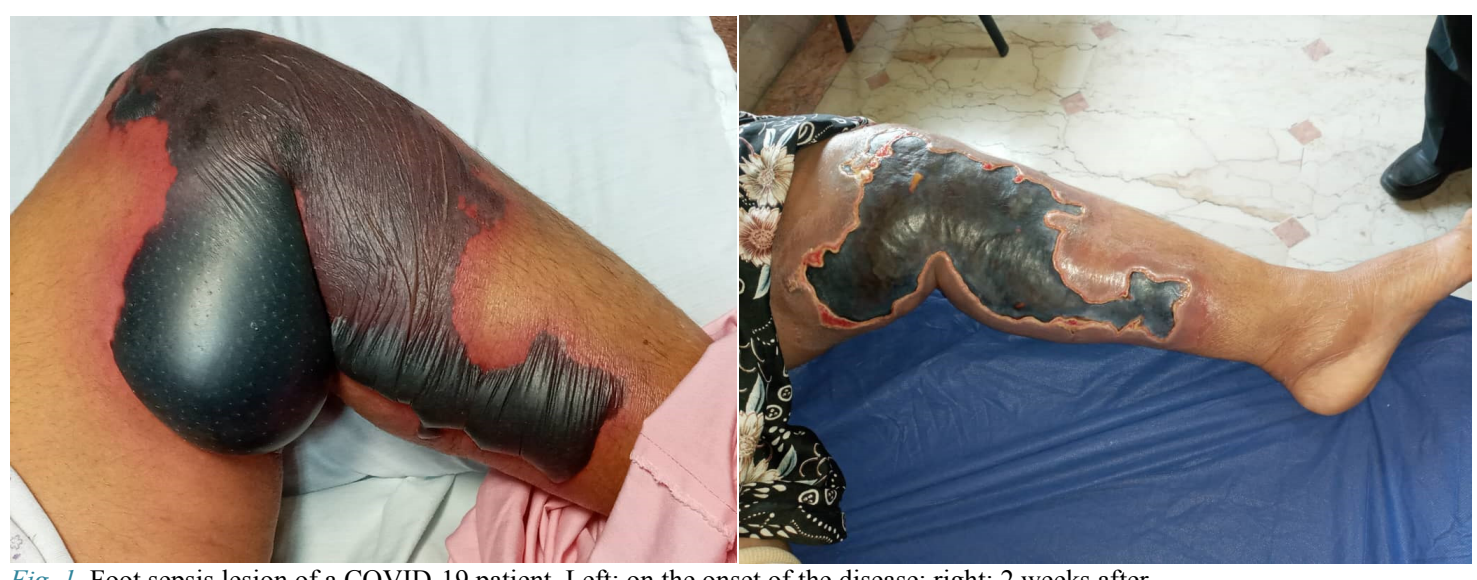

Fig. 1. Foot sepsis lesion of a COVID-19 patient. Left: on the onset of the disease; right: 2 weeks after. 

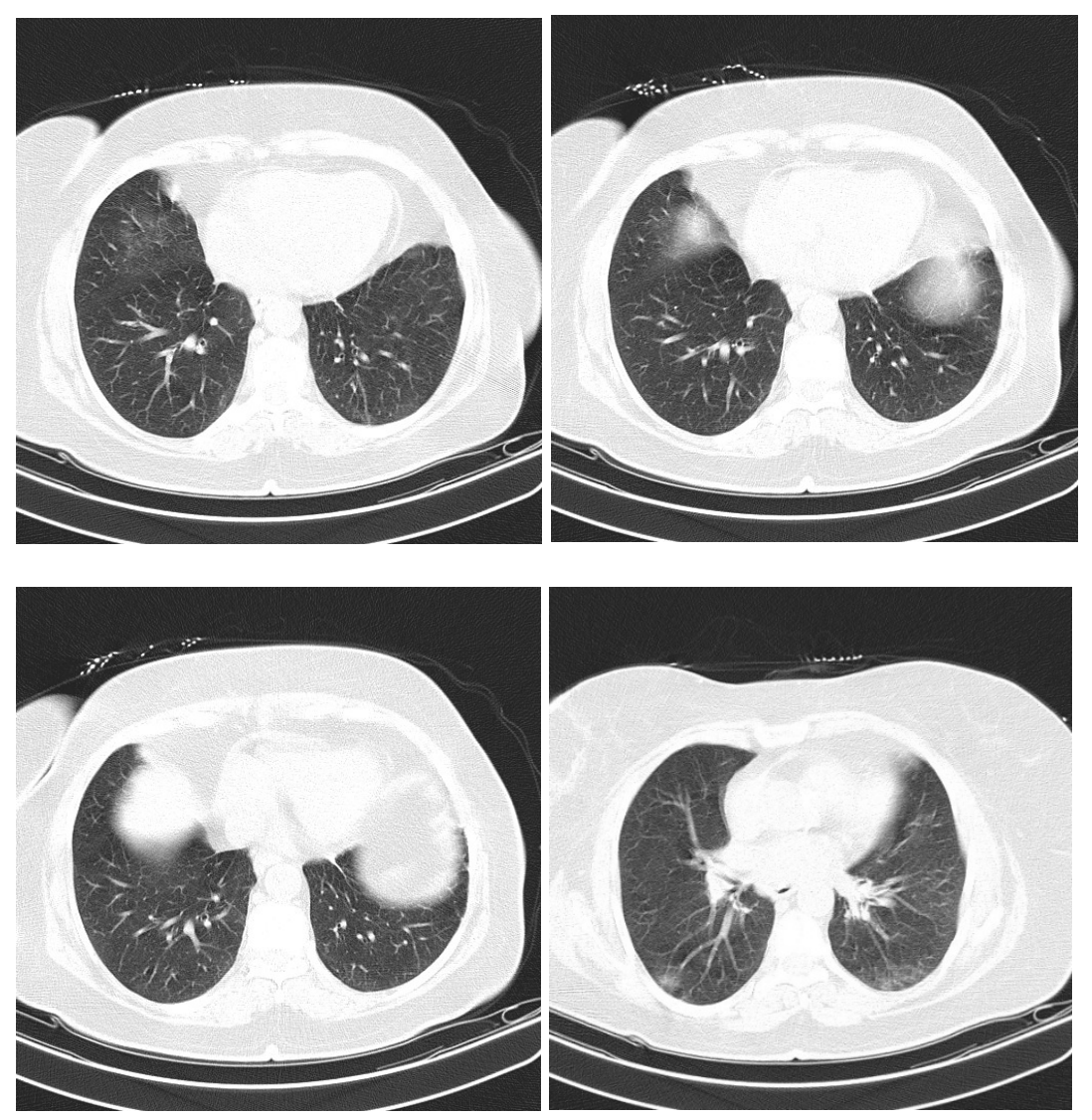

Fig. 2. Lung CT scan of the COVID-19 patient

2020, was confirmed in China (8-10), some of which had skin lesions since the beginning of the disease and some others at the time of hospitalization. Estébanez et al (11) also examined skin lesions in these patients in the study of the clinical features of COVID-19 and studied a 28-yearold woman without the previous medical history who visited the hospital with symptoms of COVID-19. The patient had erythematous plaques in the heel area that had become more severe over time. Recently, vascular injury and thrombotic events, especially in critical COVID-19 patients, have been reported in some studies, some of these patients also had cutaneous manifestations. These studies proposed the hyperactive immunological responses and cytokine storm in the pathogenesis of COVID-19 (12, 13). In the case we reported, the lesions were the same as the coagulation disorder manifestation and extensive sepsis sign, which may be produced due to immune responses to COVID-19 and a history of diabetes.

\section{Conclusion}

In conclusion, due to the very low percentage of skin symptoms in patients and their neglect and because of possibility of diagnosing them as the skin diseases as well as the very high prevalence of the disease and the possible mutations in the virus leading to various symptoms in patients, more studies are needed, so that it can help physicians to have a more accurate diagnosis of the disease.

\section{Conflict of Interests}

The authors declare that they have no competing interests.

\section{References}

1. Ahmad S, Hafeez A, Siddqui SA, Ahmad M, Mishra S. A Review of COVID-19 (Coronavirus Disease-2019) Diagnosis, Treatments and Prevention. EJMO. 2020;4(2):116-125.

2. Garg S. Hospitalization rates and characteristics of patients hospitalized with laboratory-confirmed coronavirus disease 2019COVID-NET, 14 States, March 1-30, 2020. MMWR Morb Mortal Wkly Rep. 2020 Apr 17;69(15):458-464.

3.Zhao WM, Song SH, Chen ML, Zou D, Ma LN, Ma YK, et al. The 2019 novel coronavirus resource. Hereditas. 2020;42(2):212-21.

4. Pan L, Mu M, Yang P, Sun Y, Wang R, Yan J, et al. Clinical characteristics of COVID-19 patients with digestive symptoms in Hubei, China: a descriptive, cross-sectional, multicenter study. Am J Gastroenterol. 2020 May;115(5):766-773

5. Kamali Aghdam M, Jafari N, Eftekhari K. Novel coronavirus in a 15day-old neonate with clinical signs of sepsis, a case report. Infect Dis. 2020:1-3

6. Li H, Liu L, Zhang D, Xu J, Dai H, Tang N, et al. SARS-CoV-2 and viral sepsis: observations and hypotheses. Lancet. 2020 May 9;395(10235):1517-1520

7. Recalcati S. Cutaneous manifestations in COVID-19: a first perspective. J Eur Acad Dermatol Venereol. 2020. PMID: 32215952.

8. Tang K, Wang Y, Zhang H, Zheng Q, Fang R, Sun Q. Cutaneous manifestations of the Coronavirus Disease 2019 (COVID-19): A brief review. Dermatol Ther. 2020 Jul;33(4):e13528.

9. Bogdanov I, Darlenski R, Hristakieva E, Manuelyan K. The rash that presents as a vesiculobullous eruption. Clin Dermatol. Jan-Feb 
Cutaneous hemorrhagic bullae in a patient with COVID19

2020;38(1):19-34.

10. Hunt M, Koziatek C. A Case of COVID-19 Pneumonia in a Young Male with Full Body Rash as a Presenting Symptom. Clin Pract Cases Emerg Med . 2020 May;4(2):219-221.

11. Estébanez A, Pérez-Santiago L, Silva E, Guillen-Climent S, García-Vázquez A, Ramón MD. Cutaneous manifestations in COVID-19: a new contribution. J Eur Acad Dermatol Venereol. 2020 Jun;34(6):e250-e251.

12. Seirafianpour F, Mozafarpoor S, Fattahi N, Sadeghzadeh-Bazargan A, Hanifiha M, Goodarzi A. Treatment of COVID-19 with pentoxifylline: Could it be a potential adjuvant therapy? Dermatol Ther. 2020 May 30:e13733.

13. Seirafianpour F, Sodagar S, Pour Mohammad A, Panahi P, Mozafarpoor S, Almasi S, Goodarzi A. Cutaneous manifestations and considerations in COVID-19 pandemic: A systematic review. Dermatol Ther. 2020 Jul 8:e13986. 